\title{
Robust optimization of the rate of penetration of a drill-string using a stochastic nonlinear dynamical model
}

\author{
T. G. Ritto - C. Soize • R. Sampaio
}

Received: date / Accepted: date

\begin{abstract}
This work proposes a strategy for the robust optimization of the nonlinear dynamics of a drill-string, which is a structure that rotates and digs into the rock to search for oil. The nonparametric probabilistic approach is employed to model the uncertainties of the structure as well as the uncertainties of the bit-rock interaction model. This paper is particularly concerned with the robust optimization of the rate of penetration of the column, i.e., we aim to maximize the mathematical expectation of the mean rate of penetration, respecting the integrity of the system. The variables of the optimization problem are the rotational speed at the top and the initial reaction force at the bit; they are considered deterministic. The goal is to find the set of variables that maximizes the expected mean rate of penetration, respecting, vibration limits, stress limit and fatigue limit of the dynamical system.
\end{abstract}

T. G. Ritto

Department of Mechanical Engineering, PUC-Rio, Rua Marquês de São Vicente, 225, 22453-900, Rio de Janeiro, Brazil.

Tel.: +55-21-99495644

Laboratoire de Modélisation et Simulation Multi-Echelle, MSME,

Université Paris-Est, FRE3160 CNRS, 5 bd Descartes, 77454

Marne-la-Vallée, France.

Tel.: +33-0642892769

E-mail: thiagoritto@gmail.com

C. Soize

Université Paris-Est, Laboratoire de Modélisation et Simulation Multi Echelle, MSME FRE3160 CNRS, 5 bd Descartes, 77454 Marne-la-Vallée, France.

E-mail: christian.soize@univ-paris-est.fr

R. Sampaio

Department of Mechanical Engineering, PUC-Rio, Rua Marquês de São Vicente, 225, 22453-900, Rio de Janeiro, Brazil.

E-mail: rsampaio@puc-rio.br
Keywords robust optimization · stochastic optimization · drill-string nonlinear dynamics · uncertainty modeling

\section{Introduction}

In a drilling operation, a drill-string is used to dig into the rock in search of oil. This process induces a complex dynamic response of the column (drill-string) that should be controlled to avoid accidents [1]. In addition, a drill-string robust model should take into account uncertainties, which play an important role in this problem. There are still many challenges involving the complete understanding of the nonlinear dynamics of a drillstring and the high cost of a drill-string failure justifies the interest in developing better numerical models. It should be noted that there are few works dealing with the stochastic nonlinear dynamics of a drill-string (see, $[2,3])$ and, at the best of the authors knowledge, this is the first time that a robust optimization of the dynamics of a drill-string is investigated.

The aim of this paper is to propose an optimization procedure for the nonlinear dynamics of a drillstring taken into account the uncertainties inherent in the problem. An optimization procedure that considers uncertainties is called robust optimization and its application to dynamical systems is quite recent [4-8]. In a drilling operation, the goal is to drill as fast as possible preserving the integrity of the system, i.e., avoiding failures. In the optimization strategy proposed, the objective function is the mean rate of penetration, and the constraint of the problem is its integrity limits. For the integrity limits of the structure, we use the Von Mises stress, the damage due to fatigue and a stick-slip stability factor. Fatigue is an important factor of failure in 
a drilling process $[1,9]$. The idea of this paper is to consider fatigue as a constraint to the optimization analysis without taking into account all the details (for fatigue analysis of a drill-string, see $[10,11])$. Thus, the analysis done here is more qualitative than quantitative.

There are many aspects that should be taken into account in a drilling process concerning its dynamics [12]. Some authors have proposed different models to represent the dynamics of a drill-string as, for instance, [13-21]. The model used in this paper takes into account the drive force at the top (as a constant rotational speed), the support force at the top (known as weighton-hook) and the bit-rock interaction. We consider the axial and torsional displacements of the column and the discretization is done by means of the Finite Element Method.

To model the uncertainties, the nonparametric probabilistic approach $[24,26,25,27]$ is used because (1) only one parameter is necessary to control the uncertainty of each operator of the dynamical system and (2) it takes into account both system-parameter and model uncertainties (which is important in this problem that uses simplified physical models).

The three parameters that are usually employed to control the drilling process are the rotational speed of the rotary table, the reaction force at the bottom (known as the weight-on-bit) and the fluid pump flow (less important, therefore neglected in the analysis). The value of the weight-on-bit $f_{\text {bit }}$ fluctuates; hence it would be difficult to use $f_{\text {bit }}$ in the optimization procedure. We propose then to use the initial reaction force at the bit $f_{c}$, which is used to calculate the initial prestressed state. The drilling process is stopped after every 10 meters of penetration to assemble another tube. When the operation is going to re-start, we can choose two parameters: the top speed and the static reaction force at the bit $f_{c}$ (adjusting the supporting force at the top). Then, the drilling process re-starts and the value of the $f_{\text {bit }}$ (which was initially $f_{c}$, when there was no movement of the column) now fluctuates. Therefore, the optimization variables used in the robust optimization problem are the rotational speed at the top $\omega_{\mathrm{RPM}}$ and the initial reaction force at the bit $f_{c}$.

This paper is organized as follows. The deterministic model is described in Section 2 and the probabilistic model of uncertainties is presented in Section 3. In Section 4 the robust optimization problem is presented, stating the objective function and the constraints of the problem. The numerical results are shown in Section 5 and, finally, the concluding remarks are made in Section 6 .

\section{Deterministic model}

To derive the equations of motion, the extended Hamilton Principle is applied. Defining the potential $\Pi$ by

$\Pi=\int_{t_{1}}^{t_{2}}(U-T-W) d t$,

where $U$ is the potential strain energy, $T$ is the kinetic energy and $W$ is the work done by the nonconservative forces and any force not accounted for in the potential energy. The first variation of $\Pi$ must vanish:

$\delta \Pi=\int_{t_{1}}^{t_{2}}(\delta U-\delta T-\delta W) d t=0$.

In order to focus the attention on the robust optimization problem (which is the objective of this paper), a simple nonlinear dynamical model is introduced in neglecting the lateral vibrations which are then assumed to be sufficiently small.

\subsection{Finite element discretization}

The finite element model is constructed using two-node elements with two degrees of freedom per node (axial and torsional). The finite element approximation of the displacement fields are then written as

$u(\xi, t)=\mathbf{N}_{u}(\xi) \mathbf{u}_{e}(t), \theta_{x}(\xi, t)=\mathbf{N}_{\theta_{x}}(\xi) \mathbf{u}_{e}(t)$,

where $u$ is the axial displacement, $\theta_{x}$ is the rotation about the $x$-axis, $\xi=x / l_{e}$ is the element coordinate, $\mathbf{N}$ are the shape function

$$
\begin{aligned}
& \mathbf{N}_{u}=\left[\begin{array}{llll}
(1-\xi) & 0 & \xi & 0
\end{array}\right], \\
& \mathbf{N}_{\theta_{x}}=\left[\begin{array}{llll}
0 & (1-\xi) & 0 & \xi
\end{array}\right],
\end{aligned}
$$

and

$\mathbf{u}_{e}=\left[\begin{array}{llll}u_{1} & \theta_{x 1} & u_{2} & \theta_{x 2}\end{array}\right]^{T}$,

where exponent $T$ means transposition. 
2.2 Kinetic energy

The kinetic energy is written as

$T=\frac{1}{2} \int_{0}^{L}\left(\rho A \dot{u}^{2}+\rho I_{p} \dot{\theta}_{x}^{2}\right) d x$,

where the time derivative $(d / d t)$ is denoted by a superposed dot, $\rho$ is the mass density, $A$ is the cross sectional area, $I_{p}$ is the cross sectional polar moment of inertia and $L$ is the length of the column. The first variation of the kinetic energy, after integrating by parts in time, may be written as

$\delta T=-\int_{0}^{L}\left(\rho A \ddot{u} \delta u+\rho I_{p} \ddot{\theta}_{x} \delta \theta_{x}\right) d x$,

which yields the constant mass matrix $[M]$. The element mass matrix is written as:

$[M]^{(e)}=\int_{0}^{1}\left[\rho A\left(\mathbf{N}_{u}^{T} \mathbf{N}_{u}+\rho I_{p}\left(\mathbf{N}_{\theta_{x}}^{T} \mathbf{N}_{\theta_{x}}\right)\right] l_{e} d \xi\right.$.

\subsection{Strain energy}

The strain energy is given by

$U=\frac{1}{2} \int_{V} \boldsymbol{\epsilon}^{T} \mathbf{S} d V$

where $V$ is the domain of integration, $\boldsymbol{\epsilon}=\left[\begin{array}{ll}\epsilon_{x x} & 2 \gamma_{x y}\end{array}\right.$ $\left.2 \gamma_{x z}\right]^{T}$ is the vector of the Green-Lagrange strain tensor and $\mathbf{S}$ is the vector written in the Voigt notation of the second Piola-Kirchhoff tensor. Substituting the constitutive equation $\mathbf{S}=[D] \boldsymbol{\epsilon}$ and computing the first variation of the strain energy yield

$\delta U=\int_{V} \delta \epsilon^{T}\left[\begin{array}{ccc}E & 0 & 0 \\ 0 & G & 0 \\ 0 & 0 & G\end{array}\right] \boldsymbol{\epsilon} d V$

The position $\mathbf{X}$ of the reference configuration, the position $\mathbf{x}$ of the deformed configuration, and the displacement field $\mathbf{p}$, all written in the inertial frame of reference, are such that

$\mathbf{p}=\left[\begin{array}{l}u_{x} \\ u_{y} \\ u_{z}\end{array}\right]=\mathbf{x}-\mathbf{X}=\left[\begin{array}{c}x+u \\ y \cos \left(\theta_{x}\right)-z \sin \left(\theta_{x}\right) \\ y \sin \left(\theta_{x}\right)+z \cos \left(\theta_{x}\right)\end{array}\right]-\left[\begin{array}{l}x \\ y \\ z\end{array}\right]$

then, $\left[\begin{array}{l}u_{x} \\ u_{y} \\ u_{z}\end{array}\right]=\left[\begin{array}{c}u \\ y \cos \left(\theta_{x}\right)-z \sin \left(\theta_{x}\right)-y \\ y \sin \left(\theta_{x}\right)+z \cos \left(\theta_{x}\right)-z\end{array}\right]$

Figure 1 shows that $u_{y}$ and $u_{z}$ are related to the torsion of the drill-string; the lateral displacements of the neutral line of the column are zero $(v=w=0)$.
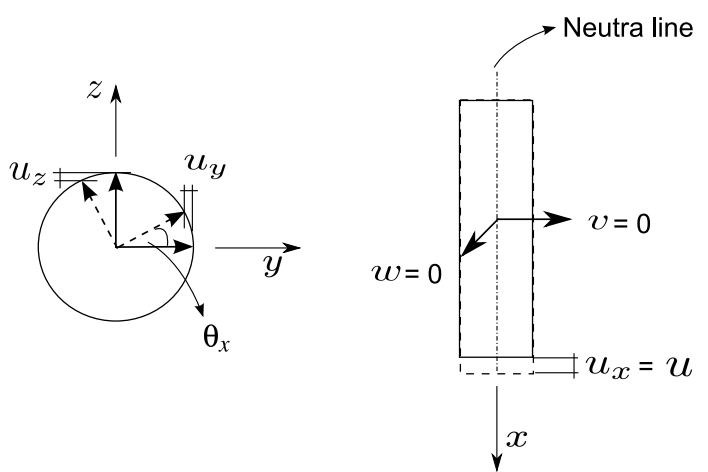

Fig. 1 Displacement field.

Finite strains are considered, thus the components of the Green-Lagrange strain tensor are written as

$$
\begin{aligned}
\epsilon_{x x} & =u_{x, x}+\frac{1}{2}\left(u_{x, x}^{2}+u_{y, x}^{2}+u_{z, x}^{2}\right), \\
\gamma_{x y} & =\frac{1}{2}\left(u_{y, x}+u_{x, y}+u_{x, x} u_{x, y}+u_{y, x} u_{y, y}+u_{z, x} u_{z, y}\right), \\
\gamma_{x z} & =\frac{1}{2}\left(u_{z, x}+u_{x, z}+u_{x, x} u_{x, z}+u_{y, x} u_{y, z}+u_{z, x} u_{z, z}\right),
\end{aligned}
$$

where $u_{x, y}=\partial u_{x} / \partial y$ and so on. Eq. (10) may be written as

$\delta U=\int_{V}\left(E \delta \epsilon_{x x} \epsilon_{x x}+4 G \delta \gamma_{x y} \gamma_{x y}+4 G \delta \gamma_{x z} \gamma_{x z}\right) d V$.

The linear terms yield the stiffness matrix $[K]$ and the higher order terms yield the geometric stiffness matrix $\left[K_{\mathrm{g}}\right]$. The element stiffness matrix is written as

$[K]^{(e)}=\int_{0}^{1}\left[\frac{E A}{l_{e}}\left(\mathbf{N}_{u}^{\prime T} \mathbf{N}_{u}^{\prime}\right)+\frac{G I_{p}}{l_{e}}\left(\mathbf{N}_{\theta_{x}}^{\prime T} \mathbf{N}_{\theta_{x}}^{\prime}\right)\right] d \xi$,

where the space derivative $(d / d \xi)$ is denoted by $\left(^{\prime}\right)$. The element geometric stiffness matrix is written as 


$$
\begin{aligned}
& {\left[K_{\mathrm{g}}\right]^{(e)}=\int_{0}^{1}\left[( \mathbf { N } _ { u } ^ { \prime T } \mathbf { N } _ { u } ^ { \prime } ) \left(3 E A u^{\prime}+1.5 E A u^{\prime 2}+\right.\right.} \\
& \left.+0.5 E I_{p} \theta_{x}^{\prime 2}\right)+\left(\mathbf{N}_{u}^{\prime T} \mathbf{N}_{\theta_{x}}^{\prime}\right)\left(E I_{p} \theta_{x}^{\prime}+E I_{p} \theta_{x}^{\prime} u^{\prime}\right)+ \\
& +\left(\mathbf{N}_{\theta_{x}}^{\prime T} \mathbf{N}_{u}^{\prime}\right)\left(E I_{p} \theta_{x}^{\prime}+E I_{p} \theta_{x}^{\prime} u^{\prime}\right)+\left(\mathbf{N}_{\theta_{x}}^{\prime T} \mathbf{N}_{\theta_{x}}^{\prime}\right)\left(E I_{p} u^{\prime}+\right. \\
& \left.\left.+0.5 E I_{p} u^{\prime 2}+1.5 E I_{p 4} \theta_{x}^{\prime 2}+3 E I_{22} \theta_{x}^{\prime 2}\right)\right] \frac{1}{l_{e}} d \xi
\end{aligned}
$$

where $u^{\prime}=\mathbf{N}_{u}^{\prime} \mathbf{u}_{e} / l_{e}, \theta_{x}^{\prime}=\mathbf{N}_{\theta_{x}}^{\prime} \mathbf{u}_{e} / l_{e}, I_{22}=\int_{A}\left(y^{2} z^{2}\right) d A$ and $I_{p 4}=\int_{A}\left(y^{4}+z^{4}\right) d A$. It should be noted that $u$ and $\theta_{x}$ are the axial displacement and the angular rotation and consequently, there are no terms related to transverse vibration in Eq. (16).

\subsection{Bit-rock interaction model}

The model used in this work for the bit-rock interaction is the one developed in [30], which can be written as

$$
\begin{aligned}
& \dot{u}_{\mathrm{bit}}=-a_{1}-a_{2} f_{\mathrm{bit}}+a_{3} \omega_{\mathrm{bit}}, \\
& t_{\mathrm{bit}}=-\frac{\dot{u}_{\mathrm{bit}}}{\omega_{\mathrm{bit}}} a_{4}-a_{5},
\end{aligned}
$$

where $f_{\text {bit }}$ is the axial force (also called weight-on-bit), $t_{\text {bit }}$ is the torque about the $x$-axis, $\dot{u}_{\text {bit }}$ is the axial speed of the bit (rate of penetration) and $\omega_{\text {bit }}$ is the rotational speed of the bit. The positive constants $a_{1}, \ldots, a_{5}$ depend on the bit and rock characteristics as well as on the average weight-on-bit. Equation (17) is rewritten as

$$
\begin{aligned}
& f_{\mathrm{bit}}=-\frac{\dot{u}_{\mathrm{bit}}}{a_{2} Z\left(\omega_{\mathrm{bit}}\right)^{2}}+\frac{a_{3} \omega_{\mathrm{bit}}}{a_{2} Z\left(\omega_{\mathrm{bit}}\right)}-\frac{a_{1}}{a_{2}}, \\
& t_{\mathrm{bit}}=-\frac{\dot{u}_{\mathrm{bit}} a_{4} Z\left(\omega_{\mathrm{bit}}\right)^{2}}{\omega_{\mathrm{bit}}}-a_{5} Z\left(\omega_{\mathrm{bit}}\right),
\end{aligned}
$$

where $e$ is the regularization parameter and $Z$ is the regularization function.

\subsection{Gravity}

The work done by gravity is written as

$$
W=\int_{0}^{L} \rho g A u d x
$$

where $g$ is the gravity acceleration. The variation of Eq. (19) gives

$\delta W=\int_{0}^{L} \rho g A \delta u d x$,

and the discretization by means of the finite element method yields the force element vector

$\mathbf{f}_{\mathrm{g}}^{(e)}=\int_{0}^{1} \mathbf{N}_{u}^{T} \rho g A l_{e} d \xi$

\subsection{Initial prestressed configuration}

Before starting the rotation about the $x$-axis, the column is put down through the channel until it reaches the soil. At this point, the forces acting on the structure are: the reaction force at the bit, the weight of the column and the supporting force at the top. In this equilibrium configuration, the column is prestressed (see Fig. 2 ). Above the neutral point the structure is tensioned and below is compressed. As it can be seen in Fig. 2, if the reaction force increases, the neutral point moves up, increasing the length of the compressed part.

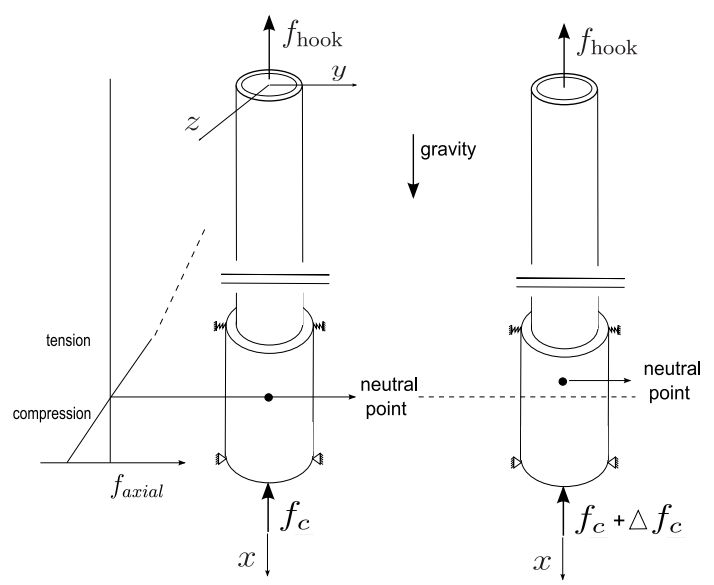

Fig. 2 Initial prestressed configuration of the system.

To calculate the initial prestressed state, the column is clamped at the top and consequently,

$\mathbf{u}_{\mathrm{S}}=[K]^{-1}\left(\mathbf{f}_{\mathrm{g}}+\mathbf{f}_{c}\right)$.

where $\mathbf{f}_{\mathrm{g}}$ is the force induced by the gravity, $\mathbf{f}_{c}$ is the vector related to the reaction force at the bit. Note that $\mathbf{f}_{c}=\left[\begin{array}{llll}0 & 0 & \ldots-f_{c} & 0\end{array}\right]^{T}$ in which $f_{c}$ is the initial reaction force at the bit. 
To verify if an element is compressed or tensioned, the element axial displacements of $\mathbf{u}_{\mathrm{S}}$ is checked. If $\left(u_{2}-u_{1}\right)>0$ the element is tensioned, if $u_{2}=u_{1}$ there is no stress and if $\left(u_{2}-u_{1}\right)<0$ the element is compressed.

\subsection{Final computational dynamical model}

Small vibrations about the initial prestressed configuration defined by $\mathbf{u}_{\mathrm{S}}$ are assumed. Therefore, the geometric stiffness matrix $\left[K_{\mathrm{g}}\left(\mathbf{u}_{\mathrm{S}}\right)\right]$ is constant. Introducing $\overline{\mathbf{u}}=\mathbf{u}-\mathbf{u}_{\mathrm{S}}$, the computational dynamical model can then be written as

$$
\begin{gathered}
{[M] \ddot{\overline{\mathbf{u}}}(t)+[C] \dot{\overline{\mathbf{u}}}(t)+\left([K]+\left[K_{\mathrm{g}}\left(\mathbf{u}_{\mathrm{S}}\right)\right]\right) \overline{\mathbf{u}}(t)=} \\
=\mathbf{g}(t)+\mathbf{f}_{\mathrm{bit}}(\dot{\overline{\mathbf{u}}}) \\
\overline{\mathbf{u}}(0)=\overline{\mathbf{u}}_{0} \quad, \quad \dot{\mathbf{u}}(0)=\overline{\mathbf{v}}_{0}
\end{gathered}
$$

in which $[M]$ and $[K]$ are the mass and stiffness matrices. The proportional damping matrix $[C]=\alpha[M]+$ $\beta[K]$ ( $\alpha$ and $\beta$ are positive constants) is added a posteriori in the computational model. The constant $\alpha$ is strictly positive. This yields that the damping matrix is positive definite although there are two rigid body modes. Such a damping model is chosen because it is assumed that there is an additional external dissipations when the dynamical system move in the two rigid body modes. The initial conditions are defined by $\overline{\mathbf{u}}_{0}$ and $\overline{\mathbf{v}}_{0}$. The force vector related to the bit-rock interaction is $\mathbf{f}_{\mathrm{bit}}$ and the imposed rotation at the top (dirichlet boundary condition) is expressed by $\mathbf{g}$.

\subsection{Reduced computational model}

To speed up the numerical simulations, the computational model is reduced in projecting the nonlinear dynamical equation on a subspace spanned by an appropriated basis. In the present paper, the basis used is made up of suitable normal modes. The normal modes are constructed from the following generalized eigenvalue problem

$$
\left([K]+\left[K_{\mathrm{g}}\left(\mathbf{u}_{\mathrm{S}}\right)\right]\right) \boldsymbol{\phi}=\omega^{2}[M] \boldsymbol{\phi}
$$

where $\phi_{i}$ is the $i$-th normal mode and $\omega_{i}$ is the corresponding natural frequency. The reduced model is written as

$$
\begin{gathered}
\overline{\mathbf{u}}(t)=[\Phi] \mathbf{q}(t), \\
{\left[M_{r}\right] \ddot{\mathbf{q}}(t)+\left[C_{r}\right] \dot{\mathbf{q}}(t)+\left[K_{r}\right] \mathbf{q}(t)=[\Phi]^{T}\left(\mathbf{g}(t)+\mathbf{f}_{\mathrm{bit}}([\Phi] \dot{\mathbf{q}})\right),} \\
\mathbf{q}(0)=\mathbf{q}_{0} \quad, \quad \dot{\mathbf{q}}(0)=\mathbf{v}_{0},
\end{gathered}
$$

in which $\mathbf{q}_{0}$ and $\mathbf{v}_{0}$ are the initial conditions and where $[\Phi]$ is the $(m \times n)$ real matrix composed by $n$ normal modes and

$$
\begin{gathered}
{\left[M_{r}\right]=[\Phi]^{T}[M][\Phi], \quad\left[C_{r}\right]=[\Phi]^{T}[C][\Phi],} \\
{\left[K_{r}\right]=[\Phi]^{T}\left([K]+\left[K_{\mathrm{g}}\left(\mathbf{u}_{\mathrm{S}}\right)\right]\right)[\Phi]}
\end{gathered}
$$

are the reduced matrices.

\section{Probabilistic model of uncertainties}

The nonparametric probabilistic approach is used to model the uncertainties in the computational model. First, the probabilistic model for the structure is presented and then the probabilistic model for the bit-rock interaction model is developed. Note that a probabilistic model that takes into account both parameter and model uncertainties is necessary to better represent the uncertainties of the problem analyzed.

\subsection{Model uncertainties for the structure}

The physical theory used to model the mechanical system (for instance, beam theory for the column) is a simplification of the real system. Therefore, it is necessary to take into account model uncertainties induced by the model errors. One way to take into account model uncertainties is to use the nonparametric probabilistic approach $[24,25,27]$ for which applications with experimental validation can be found in [31-33].

To construct the random reduced matrices, the ensembles $\mathrm{SE}^{+0}$ and $\mathrm{SE}^{+}$of random matrices defined in [25] are used. The first step is to decompose the matrices of the deterministic model applying the Cholesky decomposition

$$
\begin{aligned}
& {\left[M_{r}\right]=\left[\underline{L}_{M}\right]^{T}\left[\underline{L}_{M}\right],} \\
& {\left[C_{r}\right]=\left[\underline{L}_{C}\right]^{T}\left[\underline{L}_{C}\right]} \\
& {\left[K_{r}\right]=\left[\underline{L}_{K}\right]^{T}\left[\underline{L}_{K}\right] .}
\end{aligned}
$$

Matrices $\left[M_{r}\right],\left[C_{r}\right],\left[K_{r}\right],\left[\underline{L}_{M}\right]$ and $\left[\underline{L}_{C}\right]$ have dimension $n \times n$. The matrix $\left[\underline{L}_{K}\right]$ has dimension $p \times n$ in which $p$ is equal to $\left(n-\mu_{\text {rig }}\right)$ where $\mu_{\text {rig }}$ is the dimension of the null space of $\left[K_{r}\right]$ (note that $\mu_{\text {rig }}=2$ for the problem considered). The nonparametric probabilistic approach consists in substituting the matrices of 
the reduced deterministic model by the following three independent random matrices

$$
\begin{aligned}
& {\left[\mathbf{M}_{r}\right]=\left[\underline{L}_{M}\right]^{T}\left[\mathbf{G}_{M}\right]\left[\underline{L}_{M}\right],} \\
& {\left[\mathbf{C}_{r}\right]=\left[\underline{L}_{C}\right]^{T}\left[\mathbf{G}_{C}\right]\left[\underline{L}_{C}\right],} \\
& {\left[\mathbf{K}_{r}\right]=\left[\underline{L}_{K}\right]^{T}\left[\mathbf{G}_{K}\right]\left[\underline{\underline{L}}_{K}\right],}
\end{aligned}
$$

in which $\left[\mathbf{G}_{M}\right],\left[\mathbf{G}_{C}\right]$ and $\left[\mathbf{G}_{K}\right]$ are random matrices belonging to the ensemble $\mathrm{SE}^{+}$defined in [25]. Matrices $\left[\mathbf{G}_{M}\right]$ and $\left[\mathbf{G}_{C}\right]$ have dimension $n \times n$ and matrix $\left[\mathbf{G}_{K}\right]$ has dimension $p \times p$. The probability distribution of $\left[\mathbf{G}_{A}\right]$ (for $A \in\{M, C, K\}$ ) is completely defined and the generator of its independent realizations is given in Appendix A.

The level of statistical fluctuations of random matrix $\left[\mathbf{G}_{A}\right]$ is controlled by the dispersion parameter $\delta_{A}$ defined by

$\delta_{A}=\left\{\frac{1}{p} \mathcal{E}\left\{\left\|\left[\mathbf{G}_{A}\right]-[I]\right\|_{F}^{2}\right\}\right\}^{\frac{1}{2}}$,

where $\mathcal{E}\{\cdot\}$ denotes the mathematical expectation and $\|[A]\|_{F}=\left(\operatorname{trace}\left\{[A][A]^{T}\right\}\right)^{1 / 2}$ denotes the Frobenius norm. Consequently, the level of uncertainties for quantity $A$ is controlled by dispersion parameter $\delta_{A}$.

\subsection{Model uncertainties for the bit-rock interaction}

The probabilistic model introduced in [3] to take into account uncertainties in the bit-rock interaction forces is briefly summarized. Again, the nonparametric probabilistic approach is used to model the uncertainties. It consists in modeling the operator of the constitutive bit-rock interaction equation by a random operator. Let the generalized forces at the bit $\mathfrak{f}_{\mathrm{bit}}(\dot{\mathbf{x}}(t))$ and $\dot{\mathbf{x}}(t)$ be such that

$\mathfrak{f}_{\mathrm{bit}}(\dot{\mathbf{x}}(t))=\left(\begin{array}{c}f_{\mathrm{bit}}(\dot{\mathbf{x}}(t)) \\ t_{\mathrm{bit}}(\dot{\mathbf{x}}(t))\end{array}\right) \quad$ and $\quad \dot{\mathbf{x}}(t)=\left(\begin{array}{c}\dot{u}_{\mathrm{bit}}(t) \\ \omega_{\mathrm{bit}}(t)\end{array}\right)$

The deterministic constitutive equations of the bit-rock interaction can be written as

$\mathfrak{f}_{\mathrm{bit}}(\dot{\mathbf{x}}(t))=-\left[A_{b}(\dot{\mathbf{x}}(t))\right] \dot{\mathbf{x}}(t)$,

in which

$$
\begin{aligned}
{\left[A_{b}(\dot{\mathbf{x}}(t))\right]_{11} } & =\frac{a_{1}}{a_{2} \dot{u}_{\mathrm{bit}}(t)}+\frac{1}{a_{2} Z\left(\omega_{\mathrm{bit}}(t)\right)^{2}} \\
& -\frac{a_{3} \omega_{\mathrm{bit}}(t)}{a_{2} Z\left(\omega_{\mathrm{bit}}(t)\right) \dot{u}_{\mathrm{bit}}(t)}, \\
{\left[A_{b}(\dot{\mathbf{x}}(t))\right]_{22} } & =\frac{a_{4} Z\left(\omega_{\mathrm{bit}}(t)\right)^{2} \dot{u}_{\mathrm{bit}}(t)}{\omega_{\mathrm{bit}}(t)^{2}}+\frac{a_{5} Z\left(\omega_{\mathrm{bit}}(t)\right)}{\omega_{\mathrm{bit}}(t)}, \\
{\left[A_{b}(\dot{\mathbf{x}}(t))\right]_{12} } & =\left[A_{b}(\dot{\mathbf{x}}(t))\right]_{21}=0 .
\end{aligned}
$$

For all $\dot{\mathbf{x}}(t),\left[A_{b}(\dot{\mathbf{x}}(t))\right]$ is positive-definite. This matrix is substituted by a random matrix $\left[\mathbf{A}_{b}(\dot{\mathbf{x}}(t))\right]$ with values in the set $\mathbb{M}_{2}^{+}(\mathbb{R})$ of all the positive-definite symmetric $(2 \times 2)$ real matrices. Thus, the constitutive equation defined by Eq. (31) becomes a random constitutive equation which can be written as

$\mathfrak{F}_{\text {bit }}(\dot{\mathbf{x}}(t))=-\left[\mathbf{A}_{b}(\dot{\mathbf{x}}(t))\right] \dot{\mathbf{x}}(t)$.

The probability distribution of random variable $\left[\mathbf{A}_{b}(\dot{\mathbf{x}}(t))\right]$ is constructed for all fixed vector $\dot{\mathbf{x}}(t)$. Using the Cholesky decomposition, the mean value of $\left[A_{b}(\dot{\mathbf{x}}(t))\right]$ is written as

$\left[A_{b}(\dot{\mathbf{x}}(t))\right]=\left[L_{b}(\dot{\mathbf{x}}(t))\right]^{T}\left[L_{b}(\dot{\mathbf{x}}(t))\right]$.

The random matrix $\left[\mathbf{A}_{b}(\dot{\mathbf{x}}(t))\right]$ is defined by

$$
\left[\mathbf{A}_{b}(\dot{\mathbf{x}}(t))\right]=\left[L_{b}(\dot{\mathbf{x}}(t))\right]^{T}\left[\mathbf{G}_{b}\right]\left[L_{b}(\dot{\mathbf{x}}(t))\right],
$$

in which $\left[\mathbf{G}_{b}\right]$ belongs to the same ensemble that for $\left[\mathbf{G}_{A}\right]$ defined in the last subsection for which the dispersion parameter is defined by Eq. (29). It should be noted that, in the construction proposed, random matrix $\left[\mathbf{G}_{b}\right]$ neither depends on $\dot{\mathbf{x}}$ nor on $t$.

\subsection{Stochastic reduced computational model}

The deterministic reduced computational model defined by Eq. (25) is then replaced by the following stochastic reduced computational model

$$
\begin{gathered}
\overline{\mathbf{U}}(t)=[\Phi] \mathbf{Q}(t), \\
{\left[\mathbf{M}_{r}\right] \ddot{\mathbf{Q}}(t)+\left[\mathbf{C}_{r}\right] \dot{\mathbf{Q}}(t)+\left[\mathbf{K}_{r}\right] \mathbf{Q}(t)=} \\
=[\Phi]^{T}\left\{\mathbf{g}(t)+\mathfrak{F}_{\text {bit }}([\Phi] \dot{\mathbf{Q}}(t))\right\}, \\
\mathbf{Q}(0)=\mathbf{q}_{0}, \quad, \quad \dot{\mathbf{Q}}(0)=\mathbf{v}_{0},
\end{gathered}
$$

where $\left[\mathbf{M}_{r}\right],\left[\mathbf{K}_{r}\right]$ and $\left[\mathbf{C}_{r}\right]$ are the random matrices defined by Eq. (28), $\mathbf{Q}$ is the stochastic process of the generalized coordinates, $\overline{\mathbf{U}}$ is the stochastic process of the response of the system and $\mathfrak{F}_{\text {bit }}$ is the random force related to the bit-rock interaction probabilistic model defined by Eq. (33). 


\section{Optimization problem}

In this section, the objective function of the optimization problem is defined, the constraints related to the integrity limits of the mechanical system are presented and the robust optimization is defined.

\subsection{Objective function}

The goal of the optimization problem is to find the set of values $\mathbf{s}=\left(\omega_{\mathrm{RPM}}, f_{c}\right)$ that maximizes the expected mean rate of penetration (mean in time), respecting the integrity limits of the mechanical system. The objective function is defined by

$J(\mathbf{s})=\mathcal{E}\{\mathcal{R}(\mathbf{s})\}$,

where $J$ is the mathematical expectation of the random mean rate $\mathcal{R}$ of penetration which is such that

$\mathcal{R}(\mathbf{s})=\frac{1}{t_{1}-t_{0}} \int_{t_{0}}^{t_{1}} \dot{U}_{\mathrm{bit}}(\mathbf{s}) d t$

in which $\left(t_{0}, t_{1}\right)$ is the time interval analyzed and $\dot{U}_{\text {bit }}$ is the random rate of penetration. The constraints related to the integrity limits of the mechanical system are discussed in the next section.

\subsection{Constraints of the problem (integrity limits)}

Three constraints are proposed to represent the integrity of the mechanical system. The first one is the maximum stress value that the structure may resist. If the structure is submitted to a stress greater than the maximum admissible stress, it will fail. The second constraint is the damage cumulated by fatigue. If the damage is greater than one, a crack will occur, what is not desired. The third constraint is a stick-slip factor, since we want to avoid torsional instability and stick-slip.

The first constraint is the maximum Von Mises stress $\sigma$ (see Appendix B) that must be below the ultimate stress $\sigma_{\max }$ of the material,

$\max _{\mathbf{x}, t}\{\sigma(\mathbf{s}, \mathbf{x}, t)\} \leq \sigma_{\max }$,

where $\mathbf{x}=(x, y, z)$ belongs to the domain $\Omega_{c}$ of the problem (the column). For the stochastic problem this constraint must be true with probability $\left(1-P_{\text {risk }}\right)$.
$\operatorname{Prob}\left\{\max _{\mathbf{x}, t}\{\mathfrak{S}(\mathbf{s}, \mathbf{x}, t)\} \leq \sigma_{\max }\right\} \geq 1-P_{\text {risk }}$,

where $\mathfrak{S}$ is the random variable modeling the stress $\sigma$ in presence of uncertainties in the computational model and $P_{\text {risk }}$ represents the risk we are willing to take. The more conservative we are, the lower we set $P_{\text {risk }}$. The second constraint is the damage cumulated due to fatigue $d$ that must be below a given limit $d_{\max }$.

$\max _{\mathbf{x}}\{d(\mathbf{s}, \mathbf{x})\} \leq d_{\max }$

where $d$ is the cumulated damage related to $p_{r}$ meters of penetration. The damage $\tilde{d}$ is computed for $\left(t_{0}, t_{1}\right)$ (see Appendix C) and then, this damage is extrapolated to consider $p_{r}$ meters of penetration,

$d=\tilde{d}\left(\frac{p_{r}}{p_{d}}\right)$

where $p_{d}$ is how much it was drilled in $\left(t_{0}, t_{1}\right)$. For the stochastic problem this constraint must be true with probability $\left(1-P_{\text {risk }}\right)$.

$\operatorname{Prob}\left\{\max _{\mathbf{x}}\{D(\mathbf{s}, \mathbf{x})\} \leq d_{\max }\right\} \geq 1-P_{\text {risk }}$.

where $D(\mathbf{s}, \mathbf{x})$ is the random variable modeling $d(\mathbf{s}, \mathbf{x})$. Sometimes, in the field, engineers use a constraint related to the stick-slip instability. So, finally, the third constraint is the stick-slip stability factor $\mathfrak{s s}$ that must be below a given limit $\mathfrak{s i}_{\max }$,

$\mathfrak{s s}(\mathbf{s}) \leq \mathfrak{s s}_{\max } \cdot$

Factor $\mathfrak{s s}$ is defined by

$\mathfrak{s} \mathfrak{s}(\mathbf{s})=\frac{\omega_{b \max }(\mathbf{s})-\omega_{b \min }(\mathbf{s})}{\omega_{b \max }(\mathbf{s})+\omega_{b \min }(\mathbf{s})}$.

where $\omega_{b \max }$ is the maximum rotational speed of the bit and $\omega_{b \min }$ is the minimum rotational speed of the bit for a given time period $\left(t_{0}, t_{1}\right)$ :

$$
\begin{gathered}
\omega_{b \min }(\mathbf{s})=\min _{t \in\left(t_{0}, t_{1}\right)}\left\{\omega_{\mathrm{bit}}(\mathbf{s}, t)\right\}, \\
\omega_{b \max }(\mathbf{s})=\max _{t \in\left(t_{0}, t_{1}\right)}\left\{\omega_{\mathrm{bit}}(\mathbf{s}, t)\right\} .
\end{gathered}
$$

where $\omega_{\text {bit }}$ is the rotational speed of the bit. As the amplitude of the torsional vibrations increases, $\mathfrak{s s}$ increases, augmenting the risk of stick-slip (when $\omega_{\text {bit }}=0$ and then the bit slips). This type of oscillations must 
be avoided. For the stochastic problem this constraint must be true with probability $\left(1-P_{\text {risk }}\right)$.

$\operatorname{Prob}\left\{\mathcal{S}(\mathbf{s}) \leq \mathfrak{s s}_{\max }\right\} \geq 1-P_{\text {risk }}$,

where $\mathcal{S}(\mathbf{s})$ is the random variable modeling $\mathfrak{s}(\mathbf{s})$.

In the present analysis, the lateral displacement of the column is neglected. If lateral vibrations were taken into account, a constraint to the radial displacement $r=\sqrt{v^{2}+w^{2}}$ should be considered in which $v$ and $w$ would be the lateral displacements of the neutral line. For example, the column should have radial displacements below a given limit $r_{\max }$, thus $\max _{\mathbf{x}, t}\{r(\mathbf{s}, \mathbf{x}, t)\} \leq$ $r_{\text {max }}$. For the stochastic problem, this constraint should be true with probability $\left(1-P_{\text {risk }}\right)$, hence we would have $\operatorname{Prob}\left\{\max _{\mathbf{x}, t}\{R(\mathbf{s}, \mathbf{x}, t)\} \leq r_{\max }\right\} \geq\left(1-P_{\text {risk }}\right)$, where $R$ would be the random variable modeling $r$.

\subsection{Robust optimization problem}

The proposed robust optimization problem aims to maximize the expected mean rate of penetration of the drillstring (see Section 4.1), respecting the integrity limits of the mechanical system (see Section 4.2). It is written as

$$
\begin{array}{cc} 
& \mathbf{s}^{\mathrm{optm}}=\arg \max _{\mathbf{s} \in \mathcal{C}} J(\mathbf{s}), \\
\text { s.t. } & \operatorname{Prob}\left\{\max _{j, t}\left\{\mathfrak{S}_{j}(\mathbf{s}, t)\right\} \leq \sigma_{\max }\right\} \geq 1-P_{\text {risk }}, \\
& \operatorname{Prob}\left\{\max _{j}\left\{D_{j}(\mathbf{s})\right\} \leq d_{\max }\right\} \geq 1-P_{\text {risk }}, \\
& \operatorname{Prob}\left\{\mathcal{S}(\mathbf{s}) \leq \mathfrak{s s}_{\max }\right\} \geq 1-P_{\text {risk }},
\end{array}
$$

where the admissible set $\mathcal{C}=\left\{\mathbf{s}=\left(\omega_{\mathrm{RPM}}, f_{c}\right): \omega_{\min } \leq\right.$ $\left.\omega_{\mathrm{RPM}} \leq \omega_{\max }, f_{\min } \leq f_{c} \leq f_{\max }\right\}$. The index $j$ represents the points $\left(x_{j}, y_{j}, z_{j}\right)$ chosen for the analysis.

This robust optimization problem is not convex and it does not exist any algorithm which allows the global optimum to be surely reached with a finite number of operations. For such an optimization problem, the objective is to improve a given initial solution with an appropriate algorithm and the level of improvement got is proportional to the CPU time spent. Several techniques can be used such as random search algorithms [34] (for instance, Latin hypercube sampling type), genetic algorithms [35], local search with random restart points, etc.). Presently, since the dimension of the parameter space is small (2 parameters), a trial approach (which surely allows the initial solution to be improved) is used and is very efficient. The algorithm is then the following. A grid is generated in the parameter space and the stochastic problem is solved for each point of the grid. The points of the grid that do not satisfy the constraints of the optimization problem are eliminated. Then, the optimal point is chosen in the set of all the retained points. The identified region containing this first optimum point can be reanalyzed introducing a new refine grid around this point to improve the solution.

\section{Application of the optimization problem}

The data (geometry, material, etc.) used in the application are representative values of a drilling system, see Appendix D. The integrity limits are given by $\sigma_{\max }=$ $650 \mathrm{MPa}, d_{\max }=1$ and $\mathfrak{s i}_{\max }=1.20$. The damage $d$ and the maximum stress value $\sigma$ are calculated in the critical region of the drill-pipe, close to the drillcollar: $x=1400 \mathrm{~m}$ and $y=z=r_{0} \cos (\pi / 4)$ (where $r_{0}$ is the outer radius of the drill-pipe). The damage $d$ is calculated using $p_{r}=2000 \mathrm{~m}$, which means that we allow damage equals to one after $2000 \mathrm{~m}$ of penetration. The nonlinear dynamical system analyzed is sensitive to model uncertainties [3], therefore, the probabilistic model is fixed with $\delta_{G}=0.005$ and $\delta_{M}=\delta_{C}=\delta_{K}=$ 0.001 . The drill-string is discretized with 120 finite elements. For the construction of the reduced dynamical model, 7 torsional modes, 4 axial modes and also the two rigid body modes of the structure (axial and torsional) are used. For the time integration procedure, the implicit Newmark integration scheme has been implemented with a predictor and a fix point procedure to equilibrate the system response at each time step. All the numerical results presented below correspond to the stationary response for which the transient part of the response induced by the initial conditions has vanished, $\left(t_{0}, t_{1}\right)=(60,100) \mathrm{s}$.

\subsection{Deterministic response}

Some deterministic responses are presented in this Section. Figure 3(a) shows the axial displacement of the bit and Fig. 3(b) shows the rate of penetration for $\omega_{\mathrm{RPM}}=100 \mathrm{RPM}$ and $f_{c}=100 \mathrm{kN}$. Figure 4 shows the rotational speed of the bit for $f_{c}=100 \mathrm{kN}$, comparing $\omega_{\mathrm{RPM}}=80 \mathrm{RPM}, \omega_{\mathrm{RPM}}=120 \mathrm{RPM}$ and $\omega_{\mathrm{RPM}}=450$ RPM. No stick phase is observed (when $\omega_{\text {bit }}=0$ ), but there are significant oscillations on the rotational speed of the bit (for $\omega_{\mathrm{RPM}}=80$ and $120 \mathrm{RPM}$ ) that can be dangerous for the system, since it might cause stickslip and crack initiation due to fatigue. The dynamic response of the bit depends on the bit-rock interaction 

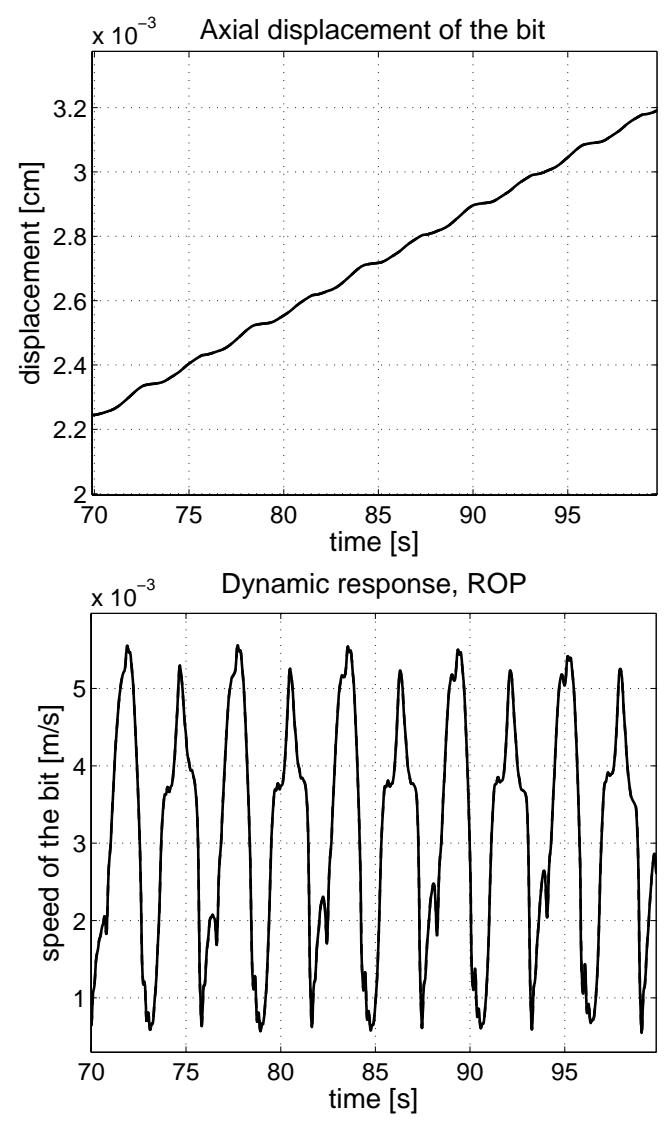

(a)

(b)

Fig. 3 (a) axial displacement of the bit and (b) rate of penetration, for $\omega_{\mathrm{RPM}}=100 \mathrm{RPM}$ and $f_{c}=100 \mathrm{kN}$.

as well as on the characteristics of the column (length, cross sectional area, material, etc). It should be noticed that for high top speeds (for example, $\omega_{\mathrm{RPM}}=450 \mathrm{RPM}$, see Fig. 4), the torque at the bit is almost constant and the oscillations of the rotational speed of the bit are small. However, as a consequence, for high top speeds the lateral vibrations (which are not considered in the analyzed model) will increase, what augment the risk of lateral instability and impacts between the column and the borehole. Figure 5 shows the force at the bit for $\omega_{\mathrm{RPM}}=100 \mathrm{RPM}$, comparing $f_{c}=100 \mathrm{kN}$ with $f_{c}=105$ $\mathrm{kN}$. Note that the force at the bit fluctuates about the value of $f_{c}$. The last result presented is the Von Misses stress for $f_{c}=100 \mathrm{kN}$ and $\omega_{\mathrm{RPM}}=100 \mathrm{RPM}$ (see Fig. 6).

In the next two sections the deterministic and the stochastic responses are going to be used to solve the deterministic and the robust optimization problem, respectively.
Dynamic response $\omega_{\text {bit }}$

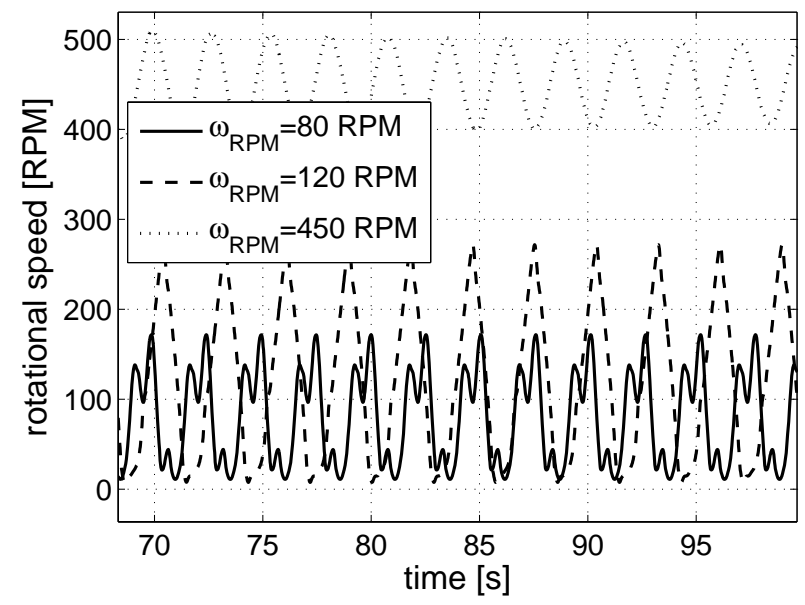

Fig. 4 Rotational speed of the bit for $f_{c}=100 \mathrm{kN}$, comparing $\omega_{\mathrm{RPM}}=80 \mathrm{RPM}, \omega_{\mathrm{RPM}}=120 \mathrm{RPM}$ and $\omega_{\mathrm{RPM}}=450 \mathrm{RPM}$.

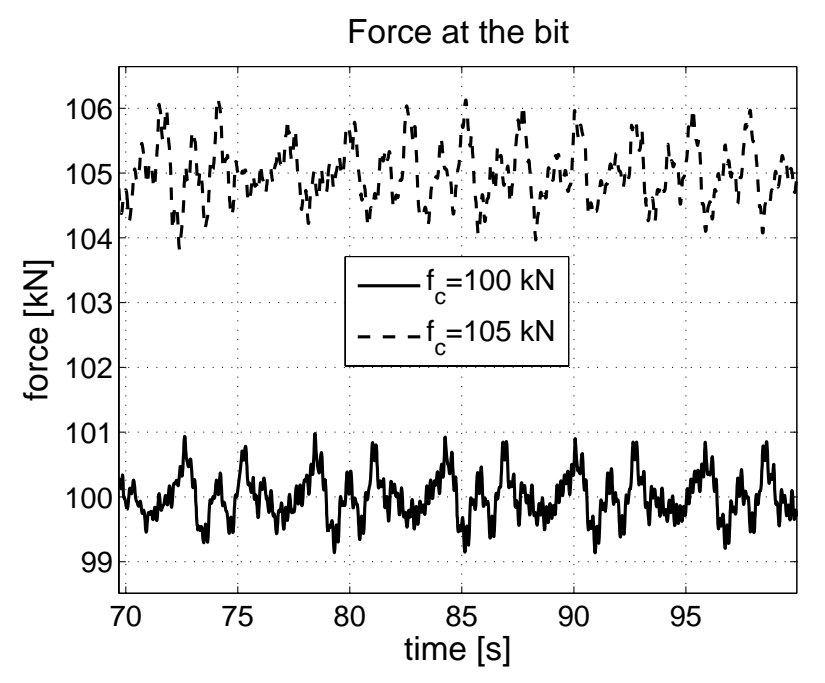

Fig. 5 Force at the bit for $\omega_{\mathrm{RPM}}=100 \mathrm{RPM}$, comparing $f_{c}=100$ $\mathrm{kN}$ and $f_{c}=105 \mathrm{kN}$.

5.2 Results of the deterministic optimization problem

In this section, the deterministic optimization problem is analyzed. For the deterministic problem, Eq. (48) is written as

$$
\mathbf{s}^{\text {optm }}=\arg \max _{\mathbf{s} \in \mathcal{C}} J^{\operatorname{det}(\mathbf{s}),}
$$

s.t. $\max _{j, t}\left\{\sigma_{j}(\mathbf{s}, t)\right\} \leq \sigma_{\max }$,

$\max _{j}\left\{d_{j}(\mathbf{s})\right\} \leq d_{\max }$,

$\mathfrak{s t}(\mathbf{s}) \leq \mathfrak{s s}_{\text {max }}$, 


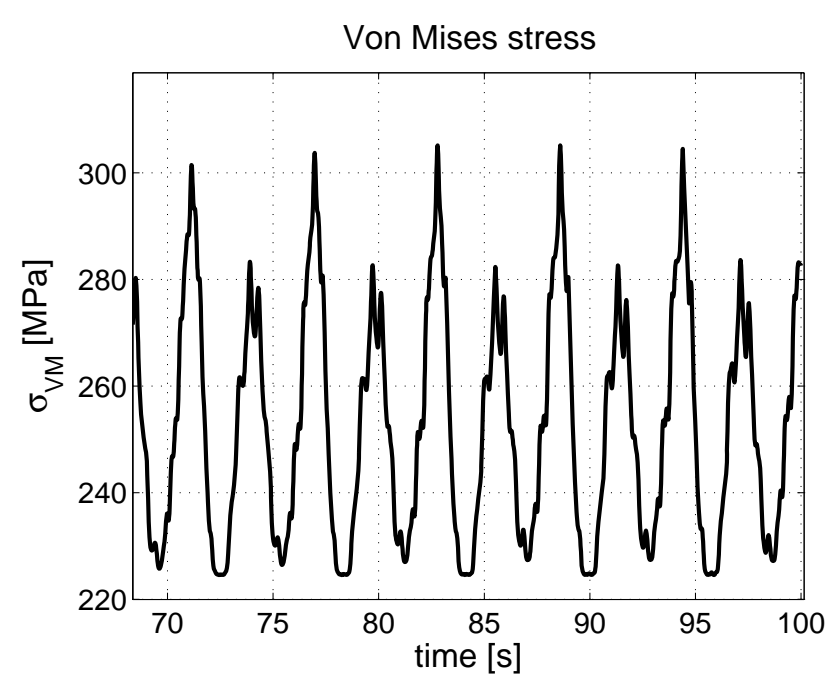

Fig. 6 Von Misses stress for $\omega_{\mathrm{RPM}}=100 \mathrm{RPM}$ and $f_{c}=100 \mathrm{kN}$

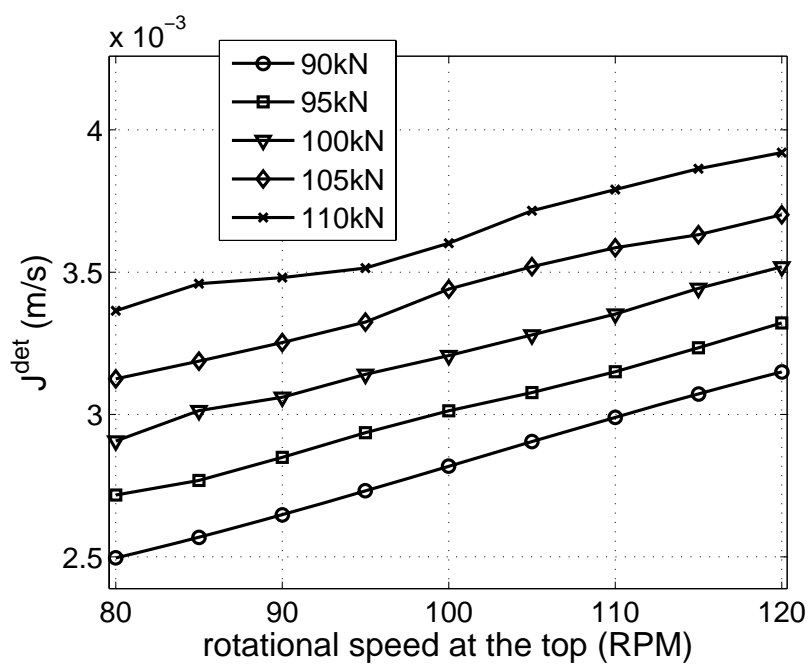

Fig. 7 Rotational speed at the top versus $J^{\text {det }}$ for different $f_{c}$ $(90,95,100,105$ and $110 \mathrm{kN})$.

where the admissible set $\mathcal{C}=\left\{\mathbf{s}=\left(\omega_{\mathrm{RPM}}, f_{c}\right): 80 R P M\right.$ $\left.\omega_{\mathrm{RPM}} \leq 120 R P M, 90 k N \leq f_{c} \leq 110 k N\right\}$ and

$J^{\operatorname{det}}(\mathbf{s})=\frac{1}{t_{1}-t_{0}} \int_{t_{0}}^{t_{1}} \dot{u}_{\mathrm{bit}}(\mathbf{s}) d t$

where $\dot{u}_{\text {bit }}$ is the deterministic rate of penetration.

Figure 7 shows the variation of $J^{\text {det }}$ with $\omega_{\mathrm{RPM}}$ for some values of $f_{c}$ which are $90,95,100,105$ and 110 $\mathrm{kN}$. When $\omega_{\mathrm{RPM}}$ and $f_{c}$ increase, $J^{\text {det }}$ also increases. Of course, there are side effects: (1) the neutral point will move upwards, (2) the column will be more flexible and (3) the dynamical response is more likely to be unstable.

To proceed with the optimization problem, we eliminate the points $\left(\omega_{\mathrm{RPM}}, f_{c}\right)$ that do not satisfy the in-

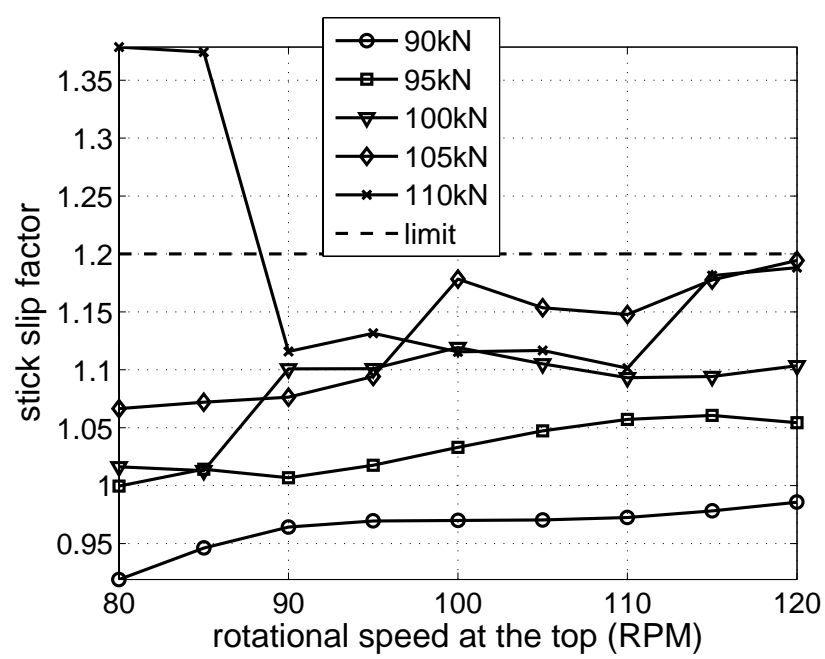

Fig. 8 Rotational speed at the top versus $\mathcal{S}_{90 \%}$ for different $f_{c}$ $(90,95,100,105$ and $110 \mathrm{kN})$. The dashed line shows the limit $\mathfrak{s i}_{\max }=1.20$.

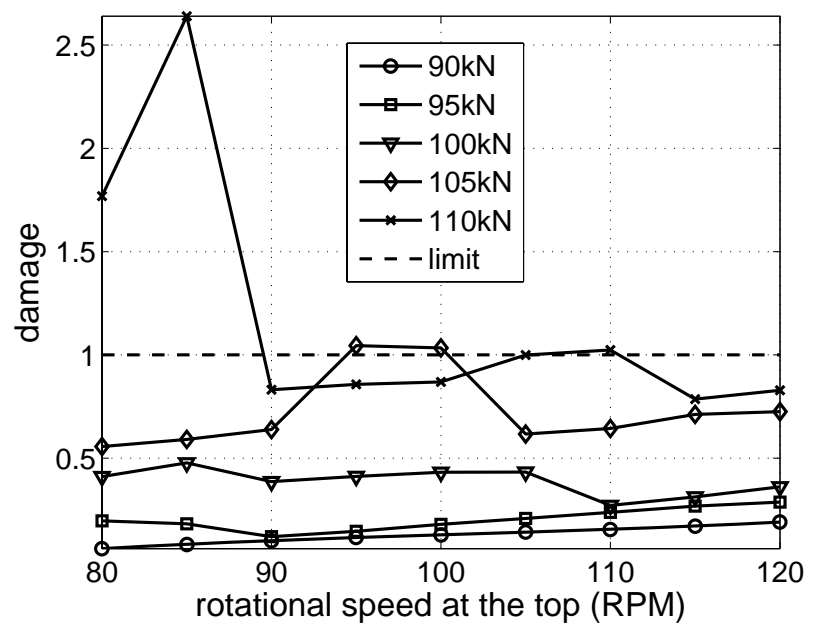

Fig. 9 Rotational speed at the top versus $D_{90 \%}$ for different $f_{c}$ $(90,95,100,105$ and $110 \mathrm{kN})$. The dashed line shows the limit $d_{\max }=1$.

tegrity limits of the system. For the points simulated, the maximum stress is always below the established limit of $\sigma_{\max }=650 \mathrm{MPa}$. Figure 8 shows $\omega_{\mathrm{RPM}}$ versus the value of the stick-slip factor $\mathfrak{s s}$ and Fig. 9 shows $\omega_{\mathrm{RPM}}$ versus the damage cumulated due to fatigue $d$ for some values of $f_{c}$. It can be seen that some points present values greater than the established limits of $\mathfrak{s i}_{\max }=1.20$ and $d_{\max }=1$.

The points that do not respect the integrity limits of the system are eliminated. Figure 10 summarizes the analysis. The points that are crossed are the ones that do not respect the constraint limits and the best point of the deterministic analysis is identified: 


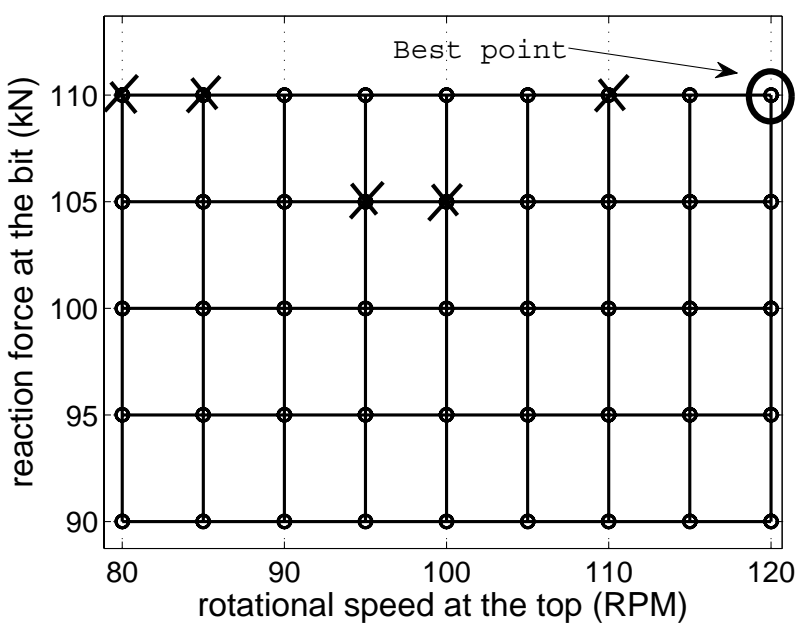

Fig. 10 Graphic showing the best point $\left(\omega_{\mathrm{RPM}}, f_{c}\right)$ (circle); the crossed points do not respect the integrity limits.

$\mathbf{s}^{\text {optm }}=\left(\omega_{\mathrm{RPM}}=120 \mathrm{RPM}, f_{c}=110 \mathrm{kN}\right)$, which gives $J_{\text {optm }}^{\text {det }}=3.92 \times 10^{-3} \mathrm{~m} / \mathrm{s} \sim 14.11 \mathrm{~m} / \mathrm{h}$.

In the next section, the results of the robust optimization problem are presented. It will be seen that the results are quite different from the ones presented in this section. The robust analysis considers the $90 \%$ percentile of the stick-slip factor, for instance. Therefore, we expect more points to be eliminated in the robust analysis, for example points $\left(\omega_{\mathrm{RPM}}=120 \mathrm{RPM}\right.$, $\left.f_{c}=105 \mathrm{kN}\right)$ and $\left(\omega_{\mathrm{RPM}}=120 \mathrm{RPM}, f_{c}=110 \mathrm{kN}\right)($ see Fig. 8), because they are already bearing the limit in the deterministic analysis.

\subsection{Results of the robust optimization problem}

Figure 11 shows the convergence of the stochastic analysis, where $\operatorname{conv}\left(n_{s}\right)=\frac{1}{n_{s}} \sum_{j=1}^{n_{s}} \int_{t_{0}}^{t_{1}}\left\|\overline{\mathbf{U}}\left(t, s_{j}\right)\right\|^{2} d t\left(n_{s}\right.$ is the number of Monte Carlo simulations).

Figure 12 shows some random realizations of the rotational speed of the bit.

Figure 13 shows the variation of $J$ with $\omega_{\mathrm{RPM}}$ for some values of $f_{c}$ which are 90, 95, 100, 105 and $110 \mathrm{kN}$. As in deterministic case, when $\omega_{\mathrm{RPM}}$ and $f_{c}$ increase, $J$ also increases, but the results are different.

To proceed with the optimization problem, we eliminate the points $\left(\omega_{\mathrm{RPM}}, f_{c}\right)$ that do not satisfy the integrity limits of the system. The constraints are considered in the analysis with $P_{\text {risk }}=10 \%$. For the points simulated, the maximum stress is always below the established limit of $\sigma_{\max }=650 \mathrm{MPa}$. Figure 14 shows $\omega_{\mathrm{RPM}}$ versus the value of the stick-slip factor $\mathcal{S}_{90 \%}$ for some values of $f_{c}$, where $\mathcal{S}_{90 \%}$ is the $90 \%$ percentile of random variable $\mathcal{S}$. Figure 15 shows $\omega_{\mathrm{RPM}}$ versus the

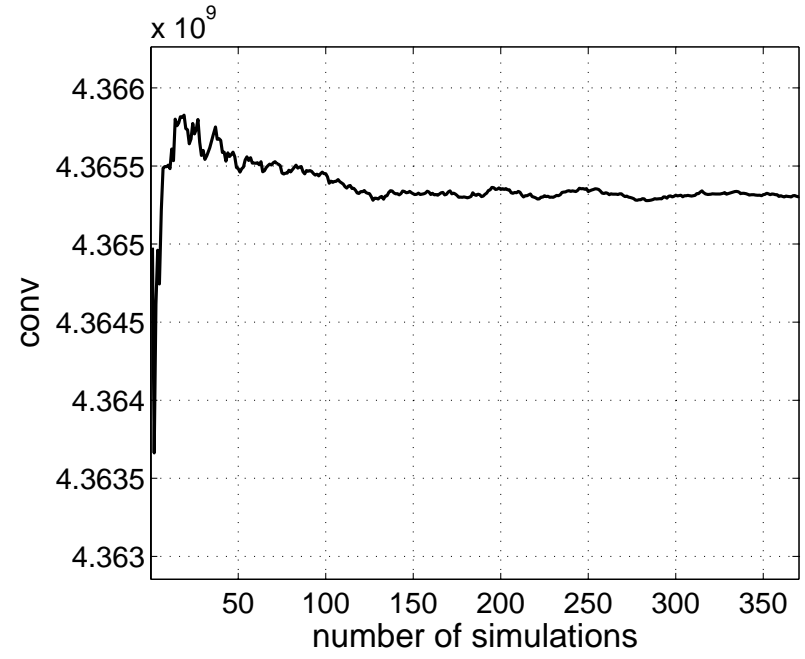

Fig. 11 Convergence function.

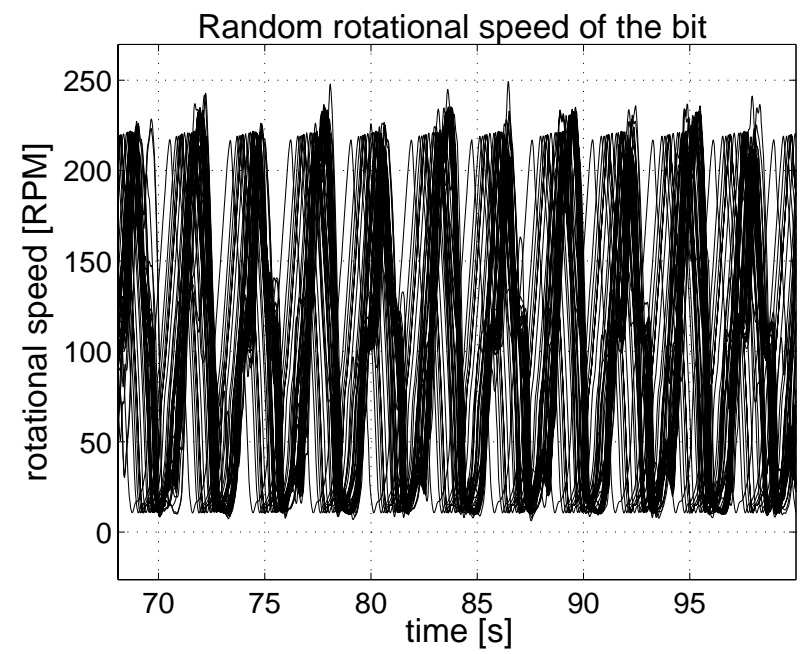

Fig. 12 Random rotation speed of the bit for $\omega_{\mathrm{RPM}}=100 \mathrm{RPM}$ and $f_{c}=100 \mathrm{kN}$.

damage cumulated due to fatigue $D_{90 \%}$, where $D_{90 \%}$ is the $90 \%$ percentile of random variable $D$. As in the deterministic analysis, it can be seen that some points present values greater than the established limits, but now there are more points in this situation.

It can be seen (Figs. 14 and 15) that the constraints are not respected for high values of $\omega_{\mathrm{RPM}}$ and $f_{c}$. Note that we would like to increase $\omega_{\mathrm{RPM}}$ and $f_{c}$ to have a higher $J$, but to respect the integrity limits these parameters are constrained.

The points that do not respect the integrity limits of the system are eliminated. Figure 16 summarizes the analysis. The points that are crossed are the ones that do not respect the constraint limits and the best point of the robust analysis is identified: $\mathbf{s}^{\mathrm{optm}}=\left(\omega_{\mathrm{RPM}}=110\right.$ 


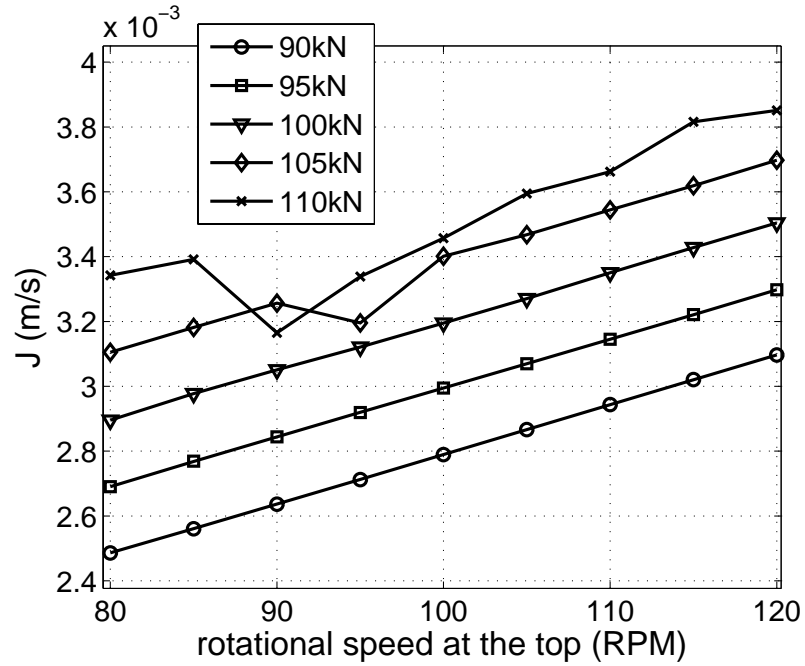

Fig. 13 Rotational speed at the top versus $J$ for different $f_{c}$ (90, $95,100,105$ and $110 \mathrm{kN})$.

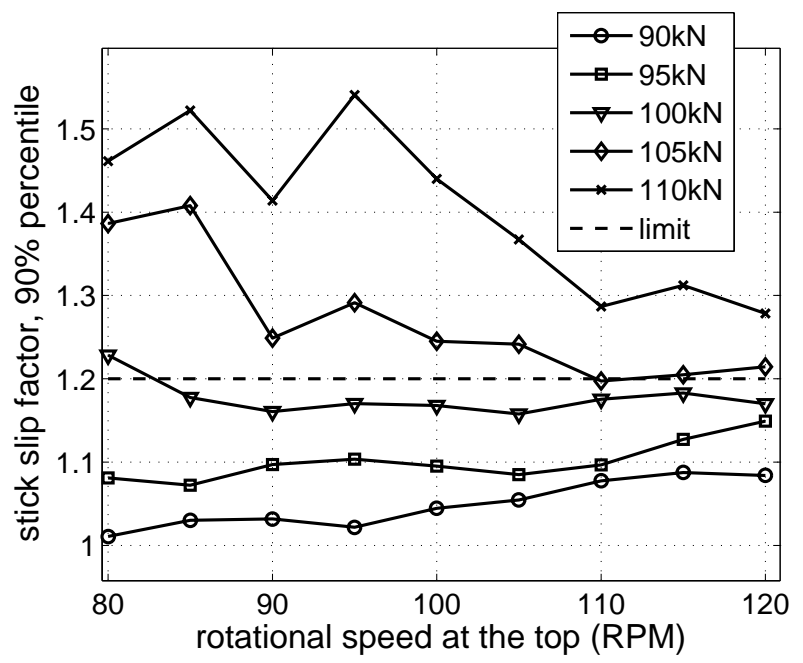

Fig. 14 Rotational speed at the top versus $\mathcal{S}_{90 \%}$ for different $f_{c}$ $(90,95,100,105$ and $110 \mathrm{kN})$. The dashed line shows the limit $\mathfrak{s s}_{\max }=1.20$.

RPM, $\left.f_{c}=105 \mathrm{kN}\right)$, which gives $J^{\text {optm }}=3.54 \times 10^{-3}$ $\mathrm{m} / \mathrm{s} \sim 12.76 \mathrm{~m} / \mathrm{h}$

It can be concluded that the robust optimization generates different results comparing to the deterministic optimization. If uncertainties are important in the dynamical analysis, we should always proceed with the robust optimization problem, instead of the deterministic optimization problem.

\section{Concluding remarks}

This paper has proposed a methodology for the robust optimization of the nonlinear dynamics of a drill-string

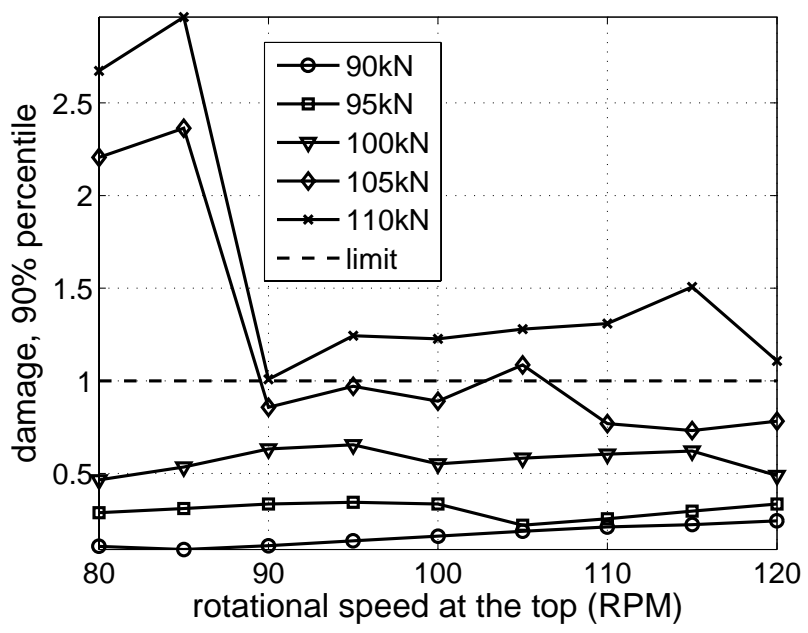

Fig. 15 Rotational speed at the top versus $D_{90 \%}$ for different $f_{c}$ $(90,95,100,105$ and $110 \mathrm{kN})$. The dashed line shows the limit $d_{\max }=1$.

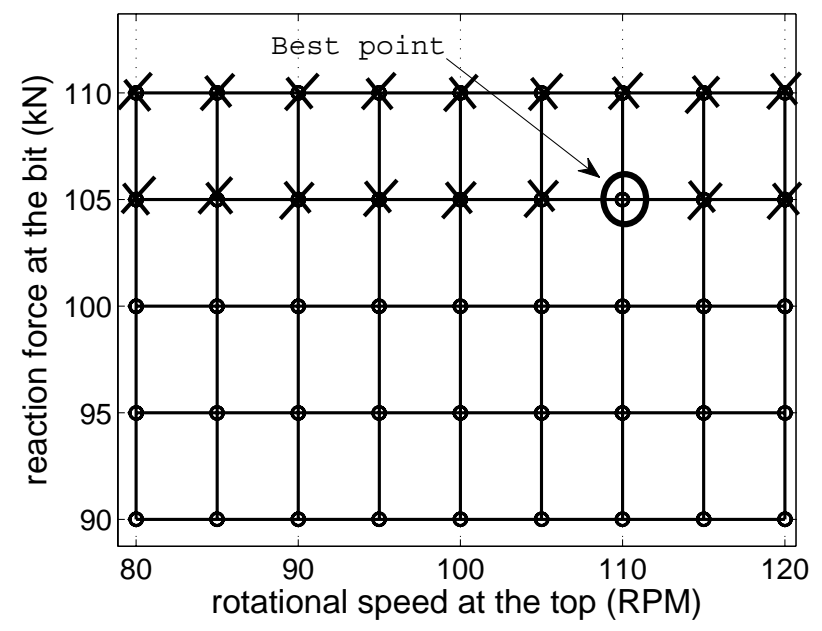

Fig. 16 Graphic showing the best point $\left(\omega_{\mathrm{RPM}}, f_{c}\right)$ (circle).

system. Applications of robust optimization in dynamical systems are quite recent. This is the first time a robust optimization problem is proposed and investigated for the nonlinear dynamics of a drill-string system. It has been shown that the robust analysis gives different results comparing to the deterministic optimization analysis. The aim of the proposed optimization problem is to maximize the expected mean rate of penetration of the drill-string, respecting the integrity limits. Three constraints have been proposed to represent the integrity of the system: (1) the ultimate stress of the material, (2) the damage cumulated by fatigue (3) a stick-slip factor.

Uncertainties are modeled using the nonparametric probabilistic approach, which takes into account both 
system-parameter and model uncertainties. This feature of the probabilistic approach used is important because a simplified mechanical model is employed in the analysis. The mass, damping and stiffness of the system, as well as the bit-rock interaction forces are considered uncertain.

The parameters of the optimization problem, which are the initial reaction force at the bit and the rotational speed at the top, have been considered deterministic. The optimization problem is solved for two cases: the computational model does not have any uncertainties and the computational model has uncertainties (robust optimization). For these two cases, the best combination of the two parameters has been found and shows that the robust optimization must be used instead of a non robust optimization.

\section{A Algorithm for the realizations of the random $\operatorname{germ}\left[\mathbf{G}_{A}\right]$}

The $p \times p$ random matrix $\left[\mathbf{G}_{A}\right]$ can be written as $\left[\mathbf{G}_{A}\right]=\left[\mathbf{L}_{A}\right]^{T}\left[\mathbf{L}_{A}\right]$ in which $\left[\mathbf{L}_{A}\right]$ is an upper triangular real random matrix such that:

1. The random variables $\left\{\left[\mathbf{L}_{A}\right]_{j j^{\prime}}, j \leq j^{\prime}\right\}$ are independents.

2. For $j<j^{\prime}$ the real-valued random variable $\left[\mathbf{L}_{A}\right]_{j j^{\prime}}=\sigma V_{j j^{\prime}}$, in which $\sigma=\delta(p+1)^{-1 / 2}$ and $V_{j j^{\prime}}$ is a real-valued gaussian random variable with zero mean and unit variance.

3. For $j=j^{\prime}$ the real-valued random variable $\left[\mathbf{L}_{A}\right]_{j j}=\sigma\left(2 V_{j}\right)^{1 / 2}$. In which $V_{j}$ is a real-valued gamma random variable whose probability density function is written as $p_{V_{j}}(v)=\mathbb{1}_{\mathbb{R}^{+}}(v) \frac{1}{\Gamma\left(\frac{p+1}{2 \delta^{2}}+\frac{1-j}{2}\right)} v^{\frac{p+1}{2 \delta^{2}}-\frac{1+j}{2}} e^{-v}$,

\section{B Stress calculation}

The numerical simulations give the axial displacement $u$ and the section area rotation $\theta_{x}$. With Eqs. (12) and (13), the displacement field and the strain are computed. Finally, the stress components are calculated doing

$$
\begin{gathered}
\sigma_{x x}=\epsilon_{x x} E, \\
\tau_{x y}=G\left(2 \gamma_{x y}\right), \\
\tau_{x z}=G\left(2 \gamma_{x z}\right) .
\end{gathered}
$$

The Von Mises stress is computed as:

$$
\sigma(t)=\sqrt{\left.\left(k_{f} \sigma_{x x}(t)\right)^{2}+3\left(\left(k_{f} \tau_{x y}(t)\right)^{2}+\left(k_{f} \tau_{x z}(t)\right)^{2}\right)\right)},
$$

where $k_{f}$ is the stress concentration factor for fatigue. The value of $k_{f}$ might vary a lot depending on several factors, such as the type of joint, tip radius, etc, [9]. In this work the value used is $k_{f}=3$.

\section{Damage calculation}

In this section we explain how the damage caused by fatigue is calculated. We use the Goodman-Wohler-Miner model.

(1) Goodman to calculate the equivalent alternate stress $\left(\sigma_{\text {eq }}\right)$ that causes a crack initiation.

$\frac{\sigma_{a}}{\sigma_{\mathrm{eq}}}+\frac{\sigma_{m}}{\sigma_{\max }}=1 \quad \longrightarrow \quad \sigma_{\mathrm{eq}}=\frac{\sigma_{a}}{1-\frac{\sigma_{m}}{\sigma_{\max }}}$

where $\sigma_{\max }$ is the ultimate stress limit of the material, $\sigma_{a}$ is the alternate Von Mises stress and $\sigma_{m}$ is the mean Von Mises stress, calculated as:

$\sigma_{a}=\frac{\max \{\sigma\}-\min \{\sigma\}}{2} \quad, \quad \sigma_{m}=\frac{\max \{\sigma\}+\min \{\sigma\}}{2}$.

(2) Wohler $\left(\right.$ or $\sigma_{\text {eq }} N$ ) to model the relationship between the stress $\left(\sigma_{\text {eq }}\right)$ and the number of cycles $(N)$ that cause a crack initiation.

$N \sigma_{\text {eq }}^{b}=c$,

where $b$ and $c$ are two positive constants that are obtained fitting experiments. We use $c=4.16 \times 10^{11}$ and $b=3$, [36]. Note that the stress value is written in $\mathrm{MPa}(b$ and $c$ will have different values for different units).

(3) Miner to calculate the damage cumulation.

$\tilde{d}=\frac{n}{N}=\frac{n}{c}\left(\sigma_{\text {eq }}\right)^{b}$.

where $n$ is the number of cycles that the structure has been subjected to.

\section{Data used in the simulation}

$L_{\mathrm{dp}}=1400 \mathrm{~m}$ (length of the drill pipe),

$L_{\mathrm{dc}}=200 \mathrm{~m}$ (length of the drill collar),

$D_{i}=0.095 \mathrm{~m}$ (inside diameter of the column),

$D_{o \mathrm{dp}}=0.12 \mathrm{~m}$ (outside diameter of the drill pipe),

$D_{o \mathrm{dc}}=0.15 \mathrm{~m}$ (outside diameter of the drill collar),

$E=210 \mathrm{GPa}$ (elasticity modulus of the drill string material),

$\rho=7850 \mathrm{~kg} / \mathrm{m}^{3}$ (density of the drill string material),

$\nu=0.29$ (poisson coefficient of the drill string material),

$g=9.81 \mathrm{~m} / \mathrm{s}^{2}$ (gravity acceleration),

$a_{1}=3.429 \times 10^{-3} \mathrm{~m} / \mathrm{s}$, (constants of the bit-rock interaction model)

$a_{2}=5.672 \times 10^{-8} \mathrm{~m} /(\mathrm{N} . \mathrm{s})$,

$a_{3}=1.374 \times 10^{-4} \mathrm{~m} / \mathrm{rd}$

$a_{4}=9.537 \times 10^{6}$ N.rd,

$a_{5}=1.475 \times 10^{3} \mathrm{~N} . \mathrm{m}$

$e=2 \mathrm{rd} / \mathrm{s}$ (regularization parameter).

The damping matrix is constructed using $[C]=\alpha[M]+\beta([K]+$ $\left.\left[K_{\mathrm{g}}\left(\mathbf{u}_{\mathrm{S}}\right)\right]\right)$ with $\alpha=0.1$ and $\beta=0.00008$.

\section{Acknowledgements}

The authors acknowledge the financial support of the Brazilian agencies CNPQ, CAPES and FAPERJ, and the French agency COFECUB (project CAPES-COFECUB 476/04). 


\section{References}

1. Macdonald KA, Bjune JV (2007) Failure analysis of drillstrings. Engineering Failure Analysis 14:1641-1666

2. Spanos PD, Chevallier AM, Politis NP (2002) Nonlinear stochastic drill-string vibrations. Journal of Vibration and Acoustics, Transactions of the ASME 124(4):512-518

3. Ritto TG, Soize C, Sampaio R (2009) Non-linear dynamics of a drill-string with uncertain model of the bit-rock interaction. International Journal of Non-Linear Mechanics 44(8):865-876

4. Ben-Tal A, Nemirovski A (2002) Robust optimization methodology and applications. Math. Programm. 92(3):453480

5. Zang C, Friswell MI, Mottershead JE (2005) A review of robust optimal design and its application in dynamics. Computers and Structures 83(4-5):315-326

6. Capiez-Lernout E, Soize C (2008) Robust design optimization in computational mechanics. Journal of Applied Mechanics, Transactions ASME 75(2):021001-1-021001-11

7. Soize C, Capiez-Lernout E, Ohayon R (2008) Robust updating of uncertain computational models using experimental modal analysis. Mechanical Systems and Signal Processing 22(8):1774-1792

8. Guedri M, Ghanmi S, Majed R, Bouhaddi N (2009) Robust tools for prediction of variability and optimization in structural dynamics. Mechanical Systems and Signal Processing 23(4):1123-1133

9. Vaisberg O, Vincke O, Perrin G, Sarda JP, Fay JB (2002) Fatigue of Drillstring: State of the Art. Oil and Gas Science and Technology 57(1):7-37

10. Bertini L, Beghini M, Santus C, Baryshnikov A (2008) Resonant test rigs for fatigue full scale testing of oil drill string connections. International Journal of Fatigue 30(6):978-988

11. Miscow GF, de Miranda PEV, Netto TA, Plácido JCR (2004) Techniques to characterize fatigue behaviour of full size drill pipes and small scale samples. International Journal of Fatigue 26:575-584

12. Spanos PD, Chevallier AM, Politis NP, Payne ML (2003) Oil and Gas Well Drilling: A Vibrations Perspective. The Shock and Vibration Digest 35(2):85-103

13. Berlioz A, Der Hagopian J, Dufour R, Draoui E (1996) Dynamic behavior of a drill-string: experimental investigation of lateral instabilities. Journal of Vibration and Accoustics 118(3):292-298

14. Christoforou AP, Yigit AS (1997) Dynamic modeling of rotating drillstrings with borehole interactions. Journal of Sound and Vibration 206(2):243-260

15. Christoforou AP, Yigit AS (2003) Fully coupled vibrations of actively controlled drillstrings. Journal of Sound and Vibration 267:1029-1045

16. Khulief YA, AL-Naser H (2005) Finite element dynamic analysis of drillstrings. Finite Elements in Analysis and Design 41:1270-1288

17. Khulief YA, Al-Sulaiman FA, Bashmal S (2007) Vibration analysis of drillstrings with self excited stick-slip oscillations. Journal of Sound and Vibration 299:540-558

18. R. Sampaio and M. T. Piovan and G. V. Lozano (2007) Coupled axial/torsional vibrations of drilling-strings by mean of nonlinear model. Mechanics Research Communications 34(56):497-502

19. Trindade MA, Wolter C, Sampaio R (2005) Karhunen-Loève decomposition of coupled axial/bending of beams subjected to impacts. Journal of Sound and Vibration 279:1015-1036

20. Tucker RW, Wang C (1999) An integrated model for drillstring dynamics. Journal of Sound and Vibration 224(1):123165
21. Yigit AS, Christoforou AP (1996) Coupled axial and transverse vibrations of oilwell drillstrings. Journal of Sound and Vibration 195(4):617-627

22. Kotsonis SJ, Spanos PD (1997) Chaotic and random whirling motion of drillstrings. Journal of Energy Resources Technology (Transactions of the ASME) 119(4):217-222

23. Spanos PD, Chevallier M (2000) Non linear stochastic drillstring vibrations. 8th ASCE Specialty Conference on Probabilistic Mechanics and Structural Reliability, South Bend, US.

24. Soize C (2000) A nonparametric model of random uncertities for reduced matrix models in structural dynamics. Probabilistic Engineering Mechanics 15:277-294

25. Soize C (2005) Random matrix theory for modeling uncertainties in computational mechanics. Computer Methods in Applied Mechanics and Engineering 194(12-16):1333-1366

26. Soize C (2005) A comprehensive overview of a nonparametric probabilistic approach of model uncertainties for predictive models in structural dynamics. Journal of Sound and Vibration 288(3):623-652

27. Soize C (2010) Generalized Probabilistic approach of uncertainties in computational dynamics using random matrices and polynomial chaos decompositions. International Journal for $\mathrm{Nu}-$ merical Methods in Engineering. Published on line 5 Aug 2009, DOI 10.1002/nme.2712.

28. Van de Vrande BL, Van Campen DH, de Kraker A (1999) An Approximate Analysis of Dry-Friction-Induced Stick-Slip Vibrations by a Smoothing Procedure. Nonlinear Dynamics 19:157-169

29. Van de Wouw N, Leine RI (2004) Attractivity of Equilibrium Sets of Systems with Dry Friction. Nonlinear Dynamics 35:1939

30. Tucker RW, Wang C (2003) Torsional vibration control and cosserat dynamics of a drill-rig assembly. Meccanica 38(1):143159

31. Chen C, Duhamel D, Soize C (2006) Probabilistic approach for model and data uncertainties and its experimental identification in structural dynamics: Case of composite sandwich panels. Journal of Sound and Vibration 194(1-2):64-81

32. Duchereau J, Soize C (2006) Transient dynamics in structures with nonhomogeneous uncertainties induced by complex joints. Mechanical Systems and Signal Processing 20:854-867

33. Durand JF, Soize C, Gagliardini L (2008) Structural-acoustic modeling of automotive vehicles in presence of uncertainties and experimental identification and validation. Journal of the Acoustical Society of America 124(3):1513-1525

34. Solis FJ, Wets RJ-B (1981) Minimization by random search techniques. Mathematics of Operations Research 6(1):19-30

35. Goldberg DE (1989) Genetic Algorithms in Search, Optimization, and Machine Learning. Addison-Wesley Professional 36. Netto TA, Lourenco MI, Botto A (2008) Fatigue performance of pre-strained pipes with girth weld defects: Full-scale experiments and analysis. International Journal of Fatigue 30:767-778 